\title{
The exterior Dirichlet problems of Monge-Ampère equations in dimension two
}

Limei Dai ${ }^{\text {* }}$

"Correspondence:

Imdai@wfu.edu.cn

1 School of Mathematics and Information Science, Weifang

University, 261061 Weifang, China

\begin{abstract}
In this paper, we study the Monge-Ampère equations $\operatorname{det} D^{2} u=f$ in dimension two with $f$ being a perturbation of $f_{0}$ at infinity. First, we obtain the necessary and sufficient conditions for the existence of radial solutions with prescribed asymptotic behavior at infinity to Monge-Ampère equations outside a unit ball. Then, using the Perron method, we get the existence of viscosity solutions with prescribed asymptotic behavior at infinity to Monge-Ampère equations outside a bounded domain.
\end{abstract}

Keywords: Monge-Ampère equations; Exterior Dirichlet problem; Asymptotic behavior

\section{Introduction}

A classical theorem for Monge-Ampère equation states that any classical convex solution of

$$
\operatorname{det} D^{2} u=1 \quad \text { in } \mathbb{R}^{n}
$$

must be a quadratic polynomial which was obtained by Jörgens [1] for $n=2$, Calabi [2] for $n \leq 5$, and Pogorelov [3] for $n \geq 2$. For $n=2$, the classical solution is either convex or concave, so the result is true without the convex hypothesis.

Cheng and Yau [4] later gave a simpler and more analytical proof of the Jörgens-CalabiPogorelov theorem along the lines of affine geometry. Caffarelli [5] extended this result to viscosity solutions. Jost and Xin [6] gave another proof of the theorem. On the other hand, it was proved by Trudinger and Wang [7] that if $D$ is an open convex subset in $\mathbb{R}^{n}$ and $u$ is a convex $C^{2}$ solution to $\operatorname{det} D^{2} u=1$ in $D$ with $\lim _{x \rightarrow \partial D} u(x)=\infty$, then $D=\mathbb{R}^{n}$.

In 2003, the Jörgens-Calabi-Pogorelov theorem was extended to exterior domains for $n \geq 2$ by Caffarelli and Li [8]. They proved that any convex viscosity solution to $\operatorname{det} D^{2} u=1$ in an exterior domain must be a quadratic polynomial at infinity for $n \geq 3$ and be the sum of a quadratic polynomial and a logarithmic term at infinity for $n=2$. That is, for $n \geq 3$, there exists a symmetric positive definite matrix $A \in \mathbb{R}^{n \times n}, \hat{b} \in \mathbb{R}^{n}, \hat{c} \in \mathbb{R}$ such that

$$
\limsup _{|x| \rightarrow \infty}|x|^{n-2}\left|u(x)-\left(\frac{1}{2} x^{T} A x+\hat{b} \cdot x+\hat{c}\right)\right|<\infty ;
$$

(c) The Author(s) 2020. This article is licensed under a Creative Commons Attribution 4.0 International License, which permits use, sharing, adaptation, distribution and reproduction in any medium or format, as long as you give appropriate credit to the original author(s) and the source, provide a link to the Creative Commons licence, and indicate if changes were made. The images or other third party material in this article are included in the article's Creative Commons licence, unless indicated otherwise in a credit line to the material. If material is not included in the article's Creative Commons licence and your intended use is not permitted by statutory regulation or exceeds the permitted use, you will need to obtain permission directly from the copyright holder. To view a copy of this licence, visit http://creativecommons.org/licenses/by/4.0/. 
and for $n=2$, there exists a symmetric positive definite matrix $A \in \mathbb{R}^{2 \times 2}, \hat{b} \in \mathbb{R}^{2}, \hat{c}, \hat{d} \in \mathbb{R}$ such that

$$
\limsup _{|x| \rightarrow \infty}|x|\left|u(x)-\left(\frac{1}{2} x^{T} A x+\hat{b} \cdot x+\hat{d} \ln \sqrt{x^{T} A x}+\hat{c}\right)\right|<\infty .
$$

For $n=2$, similar problems were studied by Ferrer, Martínez, and Milán [9, 10] applying the complex variable method. We can also refer to Delanoë [11]. Later, in [12], Caffarelli and Li also extended the Jörgens-Calabi-Pogorelov theorem to $\operatorname{det} D^{2} u=f$ in $\mathbb{R}^{n}$ with $f$ being a periodic positive function and obtained that any classical convex solution must be the sum of a quadratic polynomial and a periodic function. In 2015, Bao, Li, and Zhang [13] extended the Jörgens-Calabi-Pogorelov theorem to $\operatorname{det} D^{2} u=f$ in the exterior domain for $n \geq 2$ with $f$ being a perturbation of 1 at infinity.

The converse problem is whether the exterior Dirichlet problem has a unique solution with the prescribed asymptotic behavior. This problem was resolved, and the existence of solutions to the exterior Dirichlet problem of $\operatorname{det} D^{2} u=1$ was established in [8] for $n \geq 3$ through the Perron method. More specifically, assume that $D$ is a strictly convex and bounded domain with smooth boundary in $\mathbb{R}^{n}$ and $\phi \in C^{2}(\partial D)$, then for any symmetric positive definite matrix $A \in \mathbb{R}^{n \times n}, \hat{b} \in \mathbb{R}^{n}$, there exists a constant $c_{1}=c_{1}(n, D, \phi, \hat{b}, A)$ such that, for any $\hat{c}>c_{1}$, the exterior Dirichlet problem

$$
\begin{cases}\operatorname{det} D^{2} u=1 & \text { in } \mathbb{R}^{n} \backslash \bar{D} \\ u=\phi & \text { on } \partial D\end{cases}
$$

has a unique solution $u \in C^{\infty}\left(\mathbb{R}^{n} \backslash \bar{D}\right) \cap C^{0}\left(\overline{\mathbb{R}^{n} \backslash D}\right)$ satisfying the asymptotic behavior (1.1). For $n=2$, the existence of solutions to the exterior Dirichlet problem was established by Bao and Li [14]. More precisely, for any symmetric positive definite matrix $A \in \mathbb{R}^{2 \times 2}, \hat{b} \in$ $\mathbb{R}^{2}$, there exists a constant $d^{*}=d^{*}(D, \phi, \hat{b}, A)$ such that, for any $\hat{d}>d^{*}$, the exterior Dirichlet problem (1.3) has a unique solution $u \in C^{\infty}\left(\mathbb{R}^{2} \backslash \bar{D}\right) \cap C^{0}\left(\overline{\mathbb{R}^{2} \backslash D}\right)$ satisfying

$$
O\left(|x|^{-2}\right) \leq u(x)-\left(\frac{1}{2} x^{T} A x+\hat{b} \cdot x+\hat{d} \ln \sqrt{x^{T} A x}+c(\hat{d})\right) \leq M_{\hat{d}}+O\left(|x|^{-2}\right), \quad|x| \rightarrow \infty
$$

with $M_{\hat{d}}, c(\hat{d})$ being functions of $\hat{d}$. Bao, Li, and Zhang [13] established the existence of exterior solutions to $\operatorname{det} D^{2} u=f$ with $f$ being a perturbation of 1 at infinity for $n \geq 2$. More existence results for $n=2$ can be found in [15, 16]. In [15], global solutions and exterior solutions for Monge-Ampère equation were obtained through the new ideas in contrast to [13]. And in [16], Bao, Xiong, and Zhou proved the existence of entire solutions of Monge-Ampère equations with asymptotic behaviors employing the different method from the constructions of sub- and super- solutions. However, the existence of solutions to $\operatorname{det} D^{2} u=f$ in the exterior domain with $f$ being a perturbation of $f_{0}(|x|)$ at infinity for $n \geq 3$ was obtained by Ju and Bao [17].

The constants $\hat{c}$ and $\hat{d}$ in (1.1) and (1.2) play important roles in the existence and nonexistence of solutions to the exterior Dirichlet problem. Wang and Bao [18] studied the constants $\hat{c}$ and $\hat{d}$ among the radially symmetric solutions. They proved that, for $n \geq 3$, there exists a unique convex radial solution $u \in C^{2}\left(\mathbb{R}^{n} \backslash \overline{B_{1}(0)}\right) \cap C^{1}\left(\mathbb{R}^{n} \backslash B_{1}(0)\right)$ satisfying

$$
\operatorname{det} D^{2} u=1 \quad \text { in } \mathbb{R}^{n} \backslash \overline{B_{1}(0)} \text {, }
$$




$$
\begin{aligned}
& u=\hat{a} \quad \text { on } \partial B_{1}(0), \\
& u=\frac{1}{2}|x|^{2}+\hat{c}+O\left(|x|^{2-n}\right) \quad \text { as }|x| \rightarrow \infty,
\end{aligned}
$$

if and only if $\hat{c} \geq C^{*}=\hat{a}-\frac{1}{2}+\int_{1}^{\infty} s\left(\left(1-s^{-n}\right)^{\frac{1}{n}}-1\right) d s$; for $n=2$, there exists a unique radially symmetric solution $u \in C^{2}\left(\mathbb{R}^{2} \backslash \overline{B_{1}(0)}\right) \cap C^{1}\left(\mathbb{R}^{2} \backslash B_{1}(0)\right)$ satisfying (1.4), (1.5), and

$$
u=\frac{1}{2}|x|^{2}+\frac{\hat{d}}{2} \ln |x|+\hat{c}+O\left(|x|^{-2}\right) \quad \text { as }|x| \rightarrow \infty
$$

if and only if $\hat{d} \geq-1$ and $\hat{c}=\hat{a}+\frac{\hat{d}}{4}+\frac{\hat{d}}{2} \ln 2-\frac{1}{2}\left[(1+\hat{d})^{1 / 2}+\hat{d} \ln \left(1+(1+\hat{d})^{1 / 2}\right)\right]$. Recently, $\mathrm{Li}$ and $\mathrm{Lu}$ [19] characterized the existence and nonexistence of solutions in terms of the asymptotic behavior to the exterior Dirichlet problem with the right-hand side being 1 or the perturbation of 1 at infinity for $n \geq 3$.

In this paper, we study the Monge-Ampère equation with the right-hand side being $f_{0}(|x|)$ at infinity for $n=2$. Because of the appearance of the logarithmic term, it seems more difficult than the case of $n=3$. Assume that $f \in C^{0}\left(\mathbb{R}^{2}\right)$. Let $\beta>2$ be some constant and

$$
f(x)=f_{0}(|x|)+O\left(|x|^{-\beta}\right), \quad|x| \rightarrow \infty
$$

where $f_{0} \in C^{0}([0,+\infty))$ is positive and radially symmetric in $x$, and for some constant $\alpha$,

$$
f_{0}(|x|)=O\left(|x|^{\alpha}\right), \quad|x| \rightarrow \infty
$$

Suppose that

$$
\delta=\min \left\{-2+\frac{\alpha}{2}+\beta, 2+\frac{3}{2} \alpha\right\}>0 .
$$

Consider the Dirichlet problem outside a unit ball

$$
\begin{aligned}
& \operatorname{det} D^{2} u=f \quad \text { in } \mathbb{R}^{2} \backslash \overline{B_{1}(0)}, \\
& u=b \quad \text { on } \partial B_{1}(0) .
\end{aligned}
$$

Our first main result is the following.

Theorem 1.1 Suppose that $f$ satisfies (1.6) and $f$ is radially symmetric, that is, $f(x)=f(|x|)$. Let (1.8) hold. Then the exterior Dirichlet problem (1.9) and (1.10) has a unique convex radial solution $u \in C^{1}\left(\mathbb{R}^{2} \backslash B_{1}\right) \cap C^{2}\left(\mathbb{R}^{2} \backslash \overline{B_{1}}\right)$ satisfying, as $|x| \rightarrow \infty$,

$$
u(x)=\frac{\tilde{\tau}}{2} \int_{1}^{|x|}\left(\int_{0}^{s} 2 t f_{0}(t) d t\right)^{-\frac{1}{2}} d s+\int_{0}^{|x|}\left(\int_{0}^{s} 2 t f_{0}(t) d t\right)^{\frac{1}{2}} d s+d+O\left(|x|^{-\delta}\right)
$$

if and only if $\tilde{\tau} \geq \tilde{\tau}_{0}$ and $d=h(\tilde{\tau})$, where

$$
\tilde{\tau}_{0}:=\int_{1}^{\infty} 2 t\left(f(t)-f_{0}(t)\right) d t-\int_{0}^{1} 2 t f_{0}(t) d t
$$


and

$$
\begin{aligned}
h(\tilde{\tau})= & b-\int_{0}^{1}\left(\int_{0}^{s} 2 t f_{0}(t) d t\right)^{\frac{1}{2}} d s \\
& +\int_{1}^{\infty}\left(\int_{0}^{s} 2 t f_{0}(t) d t\right)^{\frac{1}{2}}\left[\left(1+\frac{\tilde{\tau}-\int_{s}^{\infty} 2 t\left(f(t)-f_{0}(t)\right) d t}{\int_{0}^{s} 2 t f_{0}(t) d t}\right)^{\frac{1}{2}}\right. \\
& \left.-1-\frac{1}{2} \frac{\tilde{\tau}}{\int_{0}^{s} 2 t f_{0}(t) d t}\right] d s .
\end{aligned}
$$

Remark 1.2 Let $F_{0}(x)$ denote the second term in (1.11), that is,

$$
F_{0}(x)=\int_{0}^{|x|}\left(\int_{0}^{s} 2 t f_{0}(t) d t\right)^{\frac{1}{2}} d s
$$

then $F_{0}$ satisfies

$$
\operatorname{det} D^{2} F_{0}=f_{0} \quad \text { in } \mathbb{R}^{2} \backslash\{0\} .
$$

Remark 1.3 For $n \geq 3$, the necessary and sufficient conditions of the existence of solutions with prescribed asymptotic behavior at infinity to (1.9) and (1.10) are obtained in the author's another manuscript which is submitted.

If $f \equiv 1$, the solution can be integrated. But if $f$ is general, the solution cannot be integrated directly. By extracting the corresponding quadratic polynomial and logarithmic term, we obtain the asymptotic behavior at infinity. Moreover, our approach is enough to establish the existence of solutions of the exterior Dirichlet problem. So, in the following, by constructing the sub- and super-solutions, we apply the Perron method to get the existence of solutions with prescribed asymptotic behavior at infinity to the exterior Dirichlet problem. First let us recall the definition of viscosity solutions, we can also refer to [8]. Let $O \subset \mathbb{R}^{2}$ be a domain, $g \in C^{0}(O)$ be positive, and $\Phi \in C^{0}(\partial O)$.

Definition 1.4 ([8]) Suppose that $u \in C^{0}(O)$ is locally convex. We say that $u$ is a viscosity subsolution (supersolution) of

$$
\operatorname{det} D^{2} u=g \quad \text { in } O
$$

if, for any point $\hat{x} \in O$ and any convex function $\psi \in C^{2}(O)$, whenever

$$
u(x)-\psi(x) \leq(\geq) u(\hat{x})-\psi(\hat{x})
$$

we must have

$$
\operatorname{det} D^{2} \psi(\hat{x}) \geq(\leq) g(\hat{x})
$$

$u \in C^{0}(O)$ is a viscosity solution of (1.13) if it is both a viscosity subsolution and supersolution of (1.13). 
Definition 1.5 ([8]) We say that $u \in C^{0}(O)$ is a viscosity subsolution (supersolution) of (1.13) with the boundary condition $u=\Phi$ on $\partial O$ if $u$ is a viscosity subsolution (supersolution) of (1.13), and $u \leq(\geq) \Phi$ on $\partial O$.

Then $u \in C^{0}(\bar{O})$ is a viscosity solution of (1.13) with the boundary condition $u=\Phi$ on $\partial O$ if it is a viscosity solution of (1.13) and $u=\Phi$ on $\partial O$.

Then we consider the exterior Dirichlet problem

$$
\begin{aligned}
& \operatorname{det} D^{2} u=f \quad \text { in } \mathbb{R}^{2} \backslash \bar{\Omega}, \\
& u=\varphi \quad \text { on } \partial \Omega,
\end{aligned}
$$

where $\Omega$ is a bounded domain in $\mathbb{R}^{2}, f \in C^{0}\left(\mathbb{R}^{2}\right)$ and $\varphi \in C^{2}(\partial \Omega)$. Note that here $f$ is not necessarily radially symmetric.

Let $\bar{f}(|x|), \underline{f}(|x|)$ be two positive continuous functions in $\mathbb{R}^{2}$ satisfying

$$
\bar{f}(|x|) \geq f(x) \geq f(|x|), \quad x \in \mathbb{R}^{2},
$$

and

$$
\begin{array}{ll}
f(|x|)=f_{0}(|x|)+O\left(|x|^{-\beta}\right), & |x| \rightarrow \infty, \\
\bar{f}(|x|)=f_{0}(|x|)+O\left(|x|^{-\beta}\right), & |x| \rightarrow \infty
\end{array}
$$

where $f_{0}$ is the same as (1.7). The following is the second main result.

Theorem 1.6 Let $\Omega$ be a strictly convex and bounded domain in $\mathbb{R}^{2}, 0 \in \Omega, \partial \Omega \in C^{2}$, and $\varphi \in C^{2}(\partial \Omega)$. Suppose that $f$ satisfies (1.6) and (1.8) holds. Then, for any $\tilde{b} \in \mathbb{R}^{2}$, there exists a constant $\lambda^{*}=\lambda^{*}(\tilde{b}, \varphi, f, \Omega, \alpha, \beta)$ such that, for any $\lambda>\lambda^{*}$, there exists a unique viscosity solution $u \in C^{0}\left(\mathbb{R}^{2} \backslash \Omega\right)$ to the Dirichlet problem (1.14), (1.15) which satisfies

$$
O\left(|x|^{-\delta}\right) \leq u(x)-\left(\frac{\lambda}{2} m(x)+n(x)+\tilde{b} \cdot x+\tilde{d}\right) \leq \Lambda(\lambda)+O\left(|x|^{-\delta}\right), \quad|x| \rightarrow \infty,
$$

where

$$
\begin{aligned}
& m(x)=\int_{a_{1}}^{|x|}\left(\int_{0}^{s} 2 t f_{0}(t) d t\right)^{-\frac{1}{2}} d s, \\
& n(x)=\int_{a_{1}}^{|x|}\left(\int_{0}^{s} 2 t f_{0}(t) d t\right)^{\frac{1}{2}} d s,
\end{aligned}
$$

and $a_{1}:=\min \left\{a: \Omega \subset B_{a}\right\}, B_{a}=B_{a}(0)=\left\{x \in \mathbb{R}^{2}:|x|<a\right\}, \tilde{d}$ is a function of $\lambda$, and

$$
\begin{aligned}
\Lambda(\lambda)= & \operatorname{osc}_{\partial \Omega} \varphi+\max _{\partial \Omega} \int_{|x|}^{a_{1}}\left(\int_{0}^{\infty} 2 t f_{0}(t) d t-\int_{s}^{\infty} 2 t f(t) d t+\lambda\right)^{\frac{1}{2}} d s \\
& +\int_{a_{1}}^{\infty}\left(\int_{0}^{s} 2 t f_{0}(t) d t\right)^{\frac{1}{2}}\left[\left(1+\frac{\lambda-\int_{s}^{\infty} 2 t\left(f(t)-f_{0}(t)\right) d t}{\int_{0}^{s} 2 t f_{0}(t) d t}\right)^{\frac{1}{2}}\right. \\
& \left.-\left(1+\frac{\lambda-\int_{s}^{\infty} 2 t\left(\bar{f}(t)-f_{0}(t)\right) d t}{\int_{0}^{s} 2 t f_{0}(t) d t}\right)^{\frac{1}{2}}\right] d s .
\end{aligned}
$$


Remark 1.7 If $\Omega$ is a ball, $\varphi=$ constant, and $\bar{f} \equiv f$, then $\Lambda(\lambda)=0$.

Finally, we would like to mention the blow-up solutions and asymptotic behavior of fully nonlinear equations and Monge-Ampère equations. In [20], the existence, asymptotic boundary estimates, and uniqueness of large solutions to fully nonlinear equations were studied, and in [21], the sharp conditions and asymptotic behavior of boundary blow-up solutions to the Monge-Ampère equation were studied.

This paper is organized as follows. In Sect. 2, we prove Theorem 1.1. Theorem 1.6 is proved in Sect. 3.

\section{Proof of Theorem 1.1}

Proof of Theorem 1.1 Let $u(x)=u(r)=u(|x|)$ be the radial solution to (1.9). By a direct calculation, we have

$$
\operatorname{det} D^{2} u=u^{\prime \prime} \frac{u^{\prime}}{r}=f(r), \quad r>1 .
$$

Hence

$$
\left(\left(u^{\prime}\right)^{2}\right)^{\prime}=2 r f(r), \quad r>1
$$

Then integrating the above equality from 1 to $r$ on both sides twice, we have

$$
u(r)=u(1)+\int_{1}^{r}\left(\left(u^{\prime}(1)\right)^{2}+\int_{1}^{s} 2 t f(t) d t\right)^{\frac{1}{2}} d s
$$

Let $\tau=\left(u^{\prime}(1)\right)^{2}$, and

$$
\tilde{\tau}=\int_{1}^{\infty} 2 t\left(f(t)-f_{0}(t)\right) d t+\tau-\int_{0}^{1} 2 t f_{0}(t) d t
$$

Then the exterior problem (1.9) and (1.10) has the radial solution

$$
\begin{aligned}
u(x)= & b+\int_{1}^{|x|}\left(\tau+\int_{1}^{s} 2 t f(t) d t\right)^{\frac{1}{2}} d s \\
= & b+\int_{1}^{|x|}\left(\int_{0}^{s} 2 t f_{0}(t) d t+\int_{1}^{\infty} 2 t\left(f(t)-f_{0}(t)\right) d t+\tau\right. \\
& \left.-\int_{0}^{1} 2 t f_{0}(t) d t-\int_{s}^{\infty} 2 t\left(f(t)-f_{0}(t)\right) d t\right)^{\frac{1}{2}} d s \\
= & b+\int_{1}^{|x|}\left(\int_{0}^{s} 2 t f_{0}(t) d t+\tilde{\tau}-\int_{s}^{\infty} 2 t\left(f(t)-f_{0}(t)\right) d t\right)^{\frac{1}{2}} d s \\
= & b+\int_{1}^{|x|}\left(\int_{0}^{s} 2 t f_{0}(t) d t\right)^{\frac{1}{2}}\left(1+\frac{\tilde{\tau}-\int_{s}^{\infty} 2 t\left(f(t)-f_{0}(t)\right) d t}{\int_{0}^{s} 2 t f_{0}(t) d t} d s\right. \\
= & b+\int_{1}^{|x|}\left(\int_{0}^{s} 2 t f_{0}(t) d t\right)^{\frac{1}{2}}\left\{\left[\left(1+\frac{\tilde{\tau}-\int_{s}^{\infty} 2 t\left(f(t)-f_{0}(t)\right) d t}{\int_{0}^{s} 2 t f_{0}(t) d t}\right)^{\frac{1}{2}}\right.\right.
\end{aligned}
$$




$$
\begin{aligned}
& \left.\left.-1-\frac{1}{2} \frac{\tilde{\tau}}{\int_{0}^{s} 2 t f_{0}(t) d t}\right]+1+\frac{1}{2} \frac{\tilde{\tau}}{\int_{0}^{s} 2 t f_{0}(t) d t}\right\} d s \\
= & b+\int_{1}^{|x|}\left(\int_{0}^{s} 2 t f_{0}(t) d t\right)^{\frac{1}{2}}\left[\left(1+\frac{\tilde{\tau}-\int_{s}^{\infty} 2 t\left(f(t)-f_{0}(t)\right) d t}{\int_{0}^{s} 2 t f_{0}(t) d t}\right)^{\frac{1}{2}}\right. \\
& \left.-1-\frac{1}{2} \frac{\tilde{\tau}}{\int_{0}^{s} 2 t f_{0}(t) d t}\right] d s \\
& +\frac{\tilde{\tau}}{2} \int_{1}^{|x|}\left(\int_{0}^{s} 2 t f_{0}(t) d t\right)^{-\frac{1}{2}} d s+\int_{1}^{|x|}\left(\int_{0}^{s} 2 t f_{0}(t) d t\right)^{\frac{1}{2}} d s \\
= & \frac{\tilde{\tau}}{2} \int_{1}^{|x|}\left(\int_{0}^{s} 2 t f_{0}(t) d t\right)^{-\frac{1}{2}} d s+\int_{0}^{|x|}\left(\int_{0}^{s} 2 t f_{0}(t) d t\right)^{\frac{1}{2}} d s \\
& -\int_{0}^{1}\left(\int_{0}^{s} 2 t f_{0}(t) d t\right)^{\frac{1}{2}} d s+b \\
& +\int_{1}^{\infty}\left(\int_{0}^{s} 2 t f_{0}(t) d t\right)^{\frac{1}{2}}\left[\left(1+\frac{\tilde{\tau}-\int_{s}^{\infty} 2 t\left(f(t)-f_{0}(t)\right) d t}{\int_{0}^{s} 2 t f_{0}(t) d t}\right)^{\frac{1}{2}}\right. \\
& \left.-1-\frac{1}{2} \frac{\tilde{\tau}}{\int_{0}^{s} 2 t f_{0}(t) d t}\right] d s \\
& -\int_{|x|}^{\infty}\left(\int_{0}^{s} 2 t f_{0}(t) d t\right)^{\frac{1}{2}}\left[\left(1+\frac{\tilde{\tau}-\int_{s}^{\infty} 2 t\left(f(t)-f_{0}(t)\right) d t}{\int_{0}^{s} 2 t f_{0}(t) d t}\right)^{\frac{1}{2}}\right. \\
& \left.-1-\frac{1}{2} \frac{\tilde{\tau}}{\int_{0}^{s} 2 t f_{0}(t) d t}\right] d s .
\end{aligned}
$$

Then $\tilde{\tau} \geq \tilde{\tau}_{0}$, where $\tilde{\tau}_{0}$ is the same as (1.12).

From (1.8), we know that $\alpha+2>0$. Due to (1.6) and (1.7), we have

$$
\begin{aligned}
\int_{|x|}^{\infty} & \left(\int_{0}^{s} 2 t f_{0}(t) d t\right)^{\frac{1}{2}}\left[\left(1+\frac{\tilde{\tau}-\int_{s}^{\infty} 2 t\left(f(t)-f_{0}(t)\right) d t}{\int_{0}^{s} 2 t f_{0}(t) d t}\right)^{\frac{1}{2}}\right. \\
& \left.-1-\frac{1}{2} \frac{\tilde{\tau}}{\int_{0}^{s} 2 t f_{0}(t) d t}\right] d s \\
= & O\left(|x|^{2-\frac{\alpha}{2}-\beta}\right)+O\left(|x|^{-2-\frac{3}{2} \alpha}\right) \\
= & O\left(|x|^{-\delta}\right), \quad|x| \rightarrow \infty,
\end{aligned}
$$

where $\delta>0$ is the same as (1.8).

On the other hand, if $\tilde{\tau} \geq \tilde{\tau}_{0}$, then

$$
1+\frac{\tilde{\tau}-\int_{s}^{\infty} 2 t\left(f(t)-f_{0}(t)\right) d t}{\int_{0}^{s} 2 t f_{0}(t) d t} \geq 0 .
$$

It is clear that (2.1) is a radial solution of (1.9) and (1.10) which satisfies (1.11).

If $u_{1}$ and $u_{2}$ all satisfy (1.9), (1.10), and (1.11), by the comparison principle, we know that $u_{1} \equiv u_{2}$ and obtain the uniqueness. Then we complete the proof of Theorem 1.1. 
Let

$$
\inf _{s \in[0,+\infty)} \int_{s}^{\infty} 2 t\left(f(t)-f_{0}(t)\right) d t \geq \tilde{\tau}_{0}=\int_{1}^{\infty} 2 t\left(f(t)-f_{0}(t)\right) d t-\int_{0}^{1} 2 t f_{0}(t) d t
$$

Set

$$
g(\tilde{\tau}, s)=\left(1+\frac{\tilde{\tau}-\int_{s}^{\infty} 2 t\left(f(t)-f_{0}(t)\right) d t}{\int_{0}^{s} 2 t f_{0}(t) d t}\right)^{\frac{1}{2}}-1-\frac{1}{2} \frac{\tilde{\tau}}{\int_{0}^{s} 2 t f_{0}(t) d t} .
$$

Then

$$
\frac{\partial g}{\partial \tilde{\tau}}=\frac{1}{2 \int_{0}^{s} 2 t f_{0}(t) d t}\left[\left(1+\frac{\tilde{\tau}-\int_{s}^{\infty} 2 t\left(f(t)-f_{0}(t)\right) d t}{\int_{0}^{s} 2 t f_{0}(t) d t}\right)^{-\frac{1}{2}}-1\right] .
$$

Since $\tilde{\tau} \geq \tilde{\tau}_{0}$, by (1.6) and (1.7), the infinite integral $\int_{1}^{\infty}\left(\int_{0}^{s} 2 t f_{0}(t) d t\right)^{\frac{1}{2}} \frac{\partial g}{\partial \tilde{\tau}} d s$ is convergent uniformly for $\tilde{\tau}$. So, for $h(\tilde{\tau})$ in Theorem 1.1,

$$
h^{\prime}(\tilde{\tau})=\int_{1}^{\infty}\left(\int_{0}^{s} 2 t f_{0}(t) d t\right)^{\frac{1}{2}} \frac{\partial g}{\partial \tilde{\tau}} d s .
$$

Let

$$
\tilde{\tau}^{*}=\tilde{\tau}^{*}(s)=\int_{s}^{\infty} 2 t\left(f(t)-f_{0}(t)\right) d t
$$

By (2.4), then $\tilde{\tau}^{*} \geq \tilde{\tau}_{0}$. So $h(\tilde{\tau})$ has the maximum in $\tilde{\tau}=\tilde{\tau}^{*}$ and $d=h(\tilde{\tau}) \leq h\left(\tilde{\tau}^{*}\right)$, where

$$
h\left(\tilde{\tau}^{*}\right)=b-\int_{0}^{1}\left(\int_{0}^{s} 2 t f_{0}(t) d t\right)^{\frac{1}{2}} d s-\int_{1}^{\infty} \frac{\int_{s}^{\infty} 2 t\left(f(t)-f_{0}(t)\right) d t}{2\left(\int_{0}^{s} 2 t f_{0}(t) d t\right)^{\frac{1}{2}}} d s .
$$

Hence we have the following Corollary 2.1.

Corollary 2.1 Let (2.4) hold. Then the exterior Dirichlet problem (1.9) and (1.10) has a convex radial solution $u \in C^{1}\left(\mathbb{R}^{2} \backslash B_{1}\right) \cap C^{2}\left(\mathbb{R}^{2} \backslash \overline{B_{1}}\right)$ satisfying (1.11) if and only if $\tilde{\tau} \geq \tilde{\tau}_{0}$ or $d \leq h\left(\tilde{\tau}^{*}\right)$.

If $f=f_{0} \equiv 1$, Theorem 1.1 corresponds to the results in [18]. In fact, by (2.2), we know that

$$
\begin{aligned}
u(x)= & \frac{\tilde{\tau}}{2} \int_{1}^{|x|}\left(\int_{0}^{s} 2 t d t\right)^{-\frac{1}{2}} d s+\int_{0}^{|x|}\left(\int_{0}^{s} 2 t d t\right)^{\frac{1}{2}} d s-\int_{0}^{1}\left(\int_{0}^{s} 2 t d t\right)^{\frac{1}{2}} d s \\
& +b+\int_{1}^{\infty}\left(\int_{0}^{s} 2 t d t\right)^{\frac{1}{2}}\left[\left(1+\frac{\tilde{\tau}}{\int_{0}^{s} 2 t d t}\right)^{\frac{1}{2}}-1-\frac{1}{2} \frac{\tilde{\tau}}{\int_{0}^{s} 2 t d t}\right] d s \\
& -\int_{|x|}^{\infty}\left(\int_{0}^{s} 2 t d t\right)^{\frac{1}{2}}\left[\left(1+\frac{\tilde{\tau}}{\int_{0}^{s} 2 t d t}\right)^{\frac{1}{2}}-1-\frac{1}{2} \frac{\tilde{\tau}}{\int_{0}^{s} 2 t d t}\right] d s
\end{aligned}
$$




$$
\begin{aligned}
= & \frac{\tilde{\tau}}{2} \ln |x|+\frac{1}{2}|x|^{2}-\frac{1}{2}+b+\int_{1}^{\infty} s\left[\left(1+\frac{\tilde{\tau}}{s^{2}}\right)^{\frac{1}{2}}-1-\frac{\tilde{\tau}}{2 s^{2}}\right] d s \\
& -\int_{|x|}^{\infty} s\left[\left(1+\frac{\tilde{\tau}}{s^{2}}\right)^{\frac{1}{2}}-1-\frac{\tilde{\tau}}{2 s^{2}}\right] d s .
\end{aligned}
$$

Since

$$
\begin{aligned}
& \int s\left[\left(1+\frac{\tilde{\tau}}{s^{2}}\right)^{\frac{1}{2}}-1-\frac{\tilde{\tau}}{2 s^{2}}\right] d s \\
& \quad=\frac{1}{2} s\left(s^{2}+\tilde{\tau}\right)^{\frac{1}{2}}+\frac{\tilde{\tau}}{2} \ln \left(s+\left(s^{2}+\tilde{\tau}\right)^{\frac{1}{2}}\right)-\frac{1}{2} s^{2}-\frac{\tilde{\tau}}{2} \ln s+C \quad(C \text { is any constant }), \\
& \frac{1}{2} s\left(s^{2}+\tilde{\tau}\right)^{\frac{1}{2}}=\frac{1}{2} s^{2}+\frac{\tilde{\tau}}{4}+O\left(s^{-2}\right), \quad s \rightarrow+\infty
\end{aligned}
$$

and

$$
\frac{\tilde{\tau}}{2} \ln \left(s+\left(s^{2}+\tilde{\tau}\right)^{\frac{1}{2}}\right)=\frac{\tilde{\tau}}{2} \ln s+\frac{\tilde{\tau}}{2} \ln 2+O\left(s^{-2}\right), \quad s \rightarrow+\infty
$$

then, as $|x| \rightarrow \infty$

$$
\begin{aligned}
u(x)= & \frac{\tilde{\tau}}{2} \ln |x|+\frac{1}{2}|x|^{2}+b+\frac{\tilde{\tau}}{4}+\frac{\tilde{\tau}}{2} \ln 2-\frac{1}{2}(1+\tilde{\tau})^{\frac{1}{2}} \\
& -\frac{\tilde{\tau}}{2} \ln \left(1+(1+\tilde{\tau})^{\frac{1}{2}}\right)+O\left(|x|^{-2}\right),
\end{aligned}
$$

where $\tilde{\tau}=\tau-1$. Then (2.5) corresponds to the results in [18]. In fact, in [18], $f \equiv 1,(2.1)$ can be integrated, and then the asymptotic behavior is obtained. But here we cannot integrate (2.1), and so the asymptotic behavior is more complicated.

\section{Proof of Theorem 1.6}

Proof of Theorem 1.6 By a translation, without loss of generality, we assume that $\tilde{b}=0$. Let $a_{1}:=\min \left\{a: \Omega \subset B_{a}\right\}$. Choose $a_{2}>a_{1}$. Let

$$
C_{0}=\max _{\overline{B_{a_{2}}}} f(x)>0
$$

By Lemma 3.1 in [13], for any $\xi \in \partial \Omega$, there exists a function $w_{\xi}(x)$ such that

$$
\operatorname{det} D^{2} w_{\xi}=C_{0} \quad \text { in } \mathbb{R}^{2}
$$

and $w_{\xi}(\xi)=\varphi(\xi), w_{\xi}(x)<\varphi(x)$ on $\partial \Omega \backslash\{\xi\}$.

Set

$$
w(x)=\sup _{\xi \in \partial \Omega} w_{\xi}(x), \quad x \in \mathbb{R}^{2}
$$

Then, in the viscosity sense,

$$
\operatorname{det} D^{2} w \geq C_{0} \geq f(x), \quad x \in B_{a_{2}},
$$


and

$$
w(x)=\varphi(x), \quad x \in \partial \Omega .
$$

For some constant $\lambda_{1} \geq 0$, let

$$
\underline{u}_{\lambda_{1}}(x)=\min _{\partial \Omega} \varphi+\int_{a_{1}}^{|x|}\left(\int_{1}^{s} 2 t \bar{f}(t) d t+\lambda_{1}\right)^{\frac{1}{2}} d s, \quad x \in \mathbb{R}^{2},
$$

where $\bar{f}$ is the same as (1.16). Then

$$
\operatorname{det} D^{2} \underline{u}_{\lambda_{1}}=\bar{f}(|x|), \quad x \in \mathbb{R}^{2} \backslash\{0\} .
$$

Furthermore, similar to (2.2), we have

$$
\begin{aligned}
\underline{u}_{\lambda_{1}}(x)= & \frac{\tilde{\lambda}_{1}}{2} \int_{a_{1}}^{|x|}\left(\int_{0}^{s} 2 t f_{0}(t) d t\right)^{-\frac{1}{2}} d s+\int_{a_{1}}^{|x|}\left(\int_{0}^{s} 2 t f_{0}(t) d t\right)^{\frac{1}{2}} d s+\min _{\partial \Omega} \varphi \\
& +\int_{a_{1}}^{\infty}\left(\int_{0}^{s} 2 t f_{0}(t) d t\right)^{\frac{1}{2}}\left[\left(1+\frac{\tilde{\lambda}_{1}-\int_{s}^{\infty} 2 t\left(\bar{f}(t)-f_{0}(t)\right) d t}{\int_{0}^{s} 2 t f_{0}(t) d t}\right)^{\frac{1}{2}}\right. \\
& \left.-1-\frac{1}{2} \frac{\tilde{\lambda}_{1}}{\int_{0}^{s} 2 t f_{0}(t) d t}\right] d s \\
& -\int_{|x|}^{\infty}\left(\int_{0}^{s} 2 t f_{0}(t) d t\right)^{\frac{1}{2}}\left[\left(1+\frac{\tilde{\lambda}_{1}-\int_{s}^{\infty} 2 t\left(\bar{f}(t)-f_{0}(t)\right) d t}{\int_{0}^{s} 2 t f_{0}(t) d t}\right)^{\frac{1}{2}}\right. \\
& \left.-1-\frac{1}{2} \frac{\tilde{\lambda}_{1}}{\int_{0}^{s} 2 t f_{0}(t) d t}\right] d s,
\end{aligned}
$$

where

$$
\tilde{\lambda}_{1}=\tilde{\lambda}_{1}\left(\lambda_{1}\right)=\int_{1}^{\infty} 2 t\left(\bar{f}(t)-f_{0}(t)\right) d t+\lambda_{1}-\int_{0}^{1} 2 t f_{0}(t) d t, \quad \lambda_{1} \geq 0 .
$$

In addition, similar to (2.3),

$$
\begin{aligned}
& \int_{|x|}^{\infty}\left(\int_{0}^{s} 2 t f_{0}(t) d t\right)^{\frac{1}{2}}\left[\left(1+\frac{\tilde{\lambda}_{1}-\int_{s}^{\infty} 2 t\left(\bar{f}(t)-f_{0}(t)\right) d t}{\int_{0}^{s} 2 t f_{0}(t) d t}\right)^{\frac{1}{2}}\right. \\
& \left.\quad-1-\frac{1}{2} \frac{\tilde{\lambda}_{1}}{\int_{0}^{s} 2 t f_{0}(t) d t}\right] d s \\
& \quad=O\left(|x|^{-\delta}\right), \quad|x| \rightarrow \infty,
\end{aligned}
$$

where $\delta$ is the same as (1.8). Let

$$
\begin{aligned}
v_{1}\left(\tilde{\lambda}_{1}\right)= & \min _{\partial \Omega} \varphi+\int_{a_{1}}^{\infty}\left(\int_{0}^{s} 2 t f_{0}(t) d t\right)^{\frac{1}{2}}\left[\left(1+\frac{\tilde{\lambda}_{1}-\int_{s}^{\infty} 2 t\left(\bar{f}(t)-f_{0}(t)\right) d t}{\int_{0}^{s} 2 t f_{0}(t) d t}\right)^{\frac{1}{2}}\right. \\
& \left.-1-\frac{1}{2} \frac{\tilde{\lambda}_{1}}{\int_{0}^{s} 2 t f_{0}(t) d t}\right] d s .
\end{aligned}
$$


As a result,

$$
\underline{u}_{\lambda_{1}}(x)=\frac{\tilde{\lambda}_{1}}{2} m(x)+n(x)+v_{1}\left(\tilde{\lambda}_{1}\right)+O\left(|x|^{-\delta}\right), \quad|x| \rightarrow \infty .
$$

Clearly,

$$
\underline{u}_{\lambda_{1}} \leq \min _{\partial \Omega} \varphi \leq \varphi \quad \text { on } \partial \Omega
$$

We can choose $\lambda_{1}^{*}>0$ fixed such that, for $\lambda_{1}>\lambda_{1}^{*}$,

$$
\begin{aligned}
\underline{u}_{\lambda_{1}} & =\min _{\partial \Omega} \varphi+\int_{a_{1}}^{a_{2}}\left(\int_{1}^{s} 2 t \bar{f}(t) d t+\lambda_{1}\right)^{\frac{1}{2}} d s \\
& >w(x), \quad x \in \partial B_{a_{2}} .
\end{aligned}
$$

For $\lambda_{2} \geq 0$, let

$$
\begin{aligned}
\bar{u}_{\lambda_{2}}(x)= & \max _{\partial \Omega} \varphi+\max _{\partial \Omega} \int_{|x|}^{a_{1}}\left(\int_{1}^{s} 2 t \underset{-}{f}(t) d t+\lambda_{2}\right)^{\frac{1}{2}} d s \\
& +\int_{a_{1}}^{|x|}\left(\int_{1}^{s} 2 t \underset{-}{t}(t) d t+\lambda_{2}\right)^{\frac{1}{2}} d s, \quad x \in \mathbb{R}^{2},
\end{aligned}
$$

where $f$ is the same as (1.16). Then

$$
\operatorname{det} D^{2} \bar{u}_{\lambda_{2}}=\underline{f}(|x|), \quad x \in \mathbb{R}^{2} \backslash\{0\} .
$$

Similar to (2.2), we get that

$$
\begin{aligned}
\bar{u}_{\lambda_{2}}(x)= & \frac{\tilde{\lambda}_{2}}{2} \int_{a_{1}}^{|x|}\left(\int_{0}^{s} 2 t f_{0}(t) d t\right)^{-\frac{1}{2}} d s+\int_{a_{1}}^{|x|}\left(\int_{0}^{s} 2 t f_{0}(t) d t\right)^{\frac{1}{2}} d s \\
& +\max _{\partial \Omega} \varphi+\max _{\partial \Omega} \int_{|x|}^{a_{1}}\left(\int_{1}^{s} 2 t \underline{-}(t) d t+\lambda_{2}\right)^{\frac{1}{2}} d s \\
& +\int_{a_{1}}^{\infty}\left(\int_{0}^{s} 2 t f_{0}(t) d t\right)^{\frac{1}{2}}\left[\left(1+\frac{\tilde{\lambda}_{2}-\int_{s}^{\infty} 2 t\left(f(t)-f_{0}(t)\right) d t}{\int_{0}^{s} 2 t f_{0}(t) d t}\right)^{\frac{1}{2}}\right. \\
& \left.-1-\frac{1}{2} \frac{\tilde{\lambda}_{2}}{\int_{0}^{s} 2 t f_{0}(t) d t}\right] d s \\
& -\int_{|x|}^{\infty}\left(\int_{0}^{s} 2 t f_{0}(t) d t\right)^{\frac{1}{2}}\left[\left(1+\frac{\tilde{\lambda}_{2}-\int_{s}^{\infty} 2 t\left(f(t)-f_{0}(t)\right) d t}{\int_{0}^{s} 2 t f_{0}(t) d t}\right)^{\frac{1}{2}}\right. \\
& \left.-1-\frac{1}{2} \frac{\tilde{\lambda}_{2}}{\int_{0}^{s} 2 t f_{0}(t) d t}\right] d s,
\end{aligned}
$$

where

$$
\tilde{\lambda}_{2}=\tilde{\lambda}_{2}\left(\lambda_{2}\right)=\int_{1}^{\infty} 2 t\left(f(t)-f_{0}(t)\right) d t+\lambda_{2}-\int_{0}^{1} 2 t f_{0}(t) d t, \quad \lambda_{2} \geq 0,
$$


and

$$
\begin{aligned}
& \int_{|x|}^{\infty}\left(\int_{0}^{s} 2 t f_{0}(t) d t\right)^{\frac{1}{2}}\left[\left(1+\frac{\tilde{\lambda}_{2}-\int_{s}^{\infty} 2 t\left(f(t)-f_{0}(t)\right) d t}{\int_{0}^{s} 2 t f_{0}(t) d t}\right)^{\frac{1}{2}}\right. \\
& \left.\quad-1-\frac{1}{2} \frac{\tilde{\lambda}_{2}}{\int_{0}^{s} 2 t f_{0}(t) d t}\right] d s \\
& \quad=O\left(|x|^{-\delta}\right), \quad|x| \rightarrow \infty
\end{aligned}
$$

where $\delta$ is the same as (1.8). Let

$$
\begin{aligned}
v_{2}\left(\tilde{\lambda}_{2}\right)= & \max _{\partial \Omega} \varphi+\max _{\partial \Omega} \int_{|x|}^{a_{1}}\left(\int_{1}^{s} 2 t f(t) d t+\lambda_{2}\right)^{\frac{1}{2}} d s \\
& +\int_{a_{1}}^{\infty}\left(\int_{0}^{s} 2 t f_{0}(t) d t\right)^{\frac{1}{2}}\left[\left(1+\frac{\tilde{\lambda}_{2}-\int_{s}^{\infty} 2 t\left(f(t)-f_{0}(t)\right) d t}{\int_{0}^{s} 2 t f_{0}(t) d t}\right)^{\frac{1}{2}}\right. \\
& \left.-1-\frac{1}{2} \frac{\tilde{\lambda}_{2}}{\int_{0}^{s} 2 t f_{0}(t) d t}\right] d s .
\end{aligned}
$$

As a result,

$$
\bar{u}_{\lambda_{2}}(x)=\frac{\tilde{\lambda}_{2}}{2} m(x)+n(x)+v_{2}\left(\tilde{\lambda}_{2}\right)+O\left(|x|^{-\delta}\right), \quad|x| \rightarrow \infty
$$

In addition,

$$
\begin{aligned}
\bar{u}_{\lambda_{2}}(x) & \geq \varphi+\int_{|x|}^{a_{1}}\left(\int_{1}^{s} 2 t \underset{-}{f}(t) d t+\lambda_{2}\right)^{\frac{1}{2}} d s+\int_{a_{1}}^{|x|}\left(\int_{1}^{s} 2 t \underset{-}{s}(t) d t+\lambda_{2}\right)^{\frac{1}{2}} d s \\
& =\varphi(x), \quad x \in \partial \Omega .
\end{aligned}
$$

We can choose $\lambda_{2}^{*}>0$ such that, for $\lambda_{2}>\lambda_{2}^{*}$,

$$
\begin{aligned}
\bar{u}_{\lambda_{2}}(x) & =\max _{\partial \Omega} \varphi+\int_{a_{1}}^{a_{2}}\left(\int_{1}^{s} 2 t \underset{-}{s}(t) d t+\lambda_{2}\right)^{\frac{1}{2}} d s \\
& >w(x), \quad x \in \partial B_{a_{2}} .
\end{aligned}
$$

So, by the comparison principle, we can know that

$$
w \leq \bar{u}_{\lambda_{2}} \text { in } B_{a_{2}} \backslash \Omega \text {. }
$$

Choose $\lambda^{*}>\max \left\{\tilde{\lambda}_{1}\left(\lambda_{1}^{*}\right), \tilde{\lambda}_{2}\left(\lambda_{2}^{*}\right)\right\}$ large. Then, for any $\lambda>\lambda^{*}$, we can choose appropriate constants $\lambda_{1}, \lambda_{2}$ such that

$$
\tilde{\lambda}_{1}\left(\lambda_{1}\right)=\tilde{\lambda}_{2}\left(\lambda_{2}\right)=\lambda
$$

And thus

$$
v_{1}\left(\tilde{\lambda}_{1}\right)=v_{1}(\lambda), \quad v_{2}\left(\tilde{\lambda}_{2}\right)=v_{2}(\lambda)
$$


Therefore

$$
\begin{array}{ll}
\underline{u}_{\lambda_{1}}(x)=\frac{\lambda}{2} m(x)+n(x)+v_{1}(\lambda)+O\left(|x|^{-\delta}\right), & |x| \rightarrow \infty, \\
\bar{u}_{\lambda_{2}}(x)=\frac{\lambda}{2} m(x)+n(x)+v_{2}(\lambda)+O\left(|x|^{-\delta}\right), & |x| \rightarrow \infty .
\end{array}
$$

Since $\bar{f}(t)-f_{0}(t) \geq f(t)-f_{0}(t)$, then by (3.2) and (3.5), we can deduce

$$
v_{1}(\lambda) \leq v_{2}(\lambda)
$$

From the comparison principle, we have that

$$
\underline{u}_{\lambda_{1}} \leq \bar{u}_{\lambda_{2}} \text { in } \mathbb{R}^{2} \backslash \Omega
$$

Let $\tilde{d}=v_{1}(\lambda)$, then

$$
\begin{aligned}
\underline{u}_{\lambda_{1}}(x) & =\frac{\lambda}{2} m(x)+n(x)+\tilde{d}+O\left(|x|^{-\delta}\right), \quad|x| \rightarrow \infty \\
\bar{u}_{\lambda_{2}}(x) & =\frac{\lambda}{2} m(x)+n(x)+\tilde{d}+v_{2}(\lambda)-\tilde{d}+O\left(|x|^{-\delta}\right) \\
& =\frac{\lambda}{2} m(x)+n(x)+\tilde{d}+\Lambda(\lambda)+O\left(|x|^{-\delta}\right), \quad|x| \rightarrow \infty
\end{aligned}
$$

where $\Lambda(\lambda)$ is the same as (1.17). Define

$$
\underline{u}(x)= \begin{cases}\max \left\{w(x), \underline{u}_{\lambda_{1}}(x)\right\}, & x \in B_{a_{2}} \backslash \Omega, \\ \underline{u}_{\lambda_{1}}(x), & x \in \mathbb{R}^{2} \backslash B_{a_{2}} .\end{cases}
$$

Then $\underline{u} \in C^{0}\left(\mathbb{R}^{2} \backslash \Omega\right)$ satisfies

$$
\operatorname{det} D^{2} \underline{u} \geq f \quad \text { in } \mathbb{R}^{2} \backslash \bar{\Omega},
$$

and by (3.1) and (3.3),

$$
\underline{u}=w=\varphi \quad \text { on } \partial \Omega .
$$

By (3.7) and (3.8),

$$
\underline{u} \leq \bar{u}_{\lambda_{2}} \quad \text { in } \mathbb{R}^{2} \backslash \Omega .
$$

Let $\mathbb{S}_{\tilde{d}}$ denote the set of subsolutions $v$ to the Dirichlet problem (1.14), (1.15) satisfying

$$
v(x) \leq \bar{u}_{\lambda_{2}}(x), \quad x \in \mathbb{R}^{2} \backslash \Omega
$$

Then $\underline{u} \in \mathbb{S}_{\tilde{d}}$. Define

$$
u(x)=\sup \left\{v(x): v \in \mathbb{S}_{\tilde{d}}\right\}
$$


So

$$
\underline{u}(x) \leq u(x) \leq \bar{u}_{\lambda_{2}}(x), \quad x \in \mathbb{R}^{2} \backslash \Omega .
$$

Thus, by (3.9) and (3.10), we know that

$$
O\left(|x|^{-\delta}\right) \leq u(x)-\left(\frac{\lambda}{2} m(x)+n(x)+\tilde{d}\right) \leq \Lambda(\lambda)+O\left(|x|^{-\delta}\right), \quad|x| \rightarrow \infty .
$$

Moreover, $u$ is a viscosity subsolution of (1.14). By (3.11), for any $x_{0} \in \partial \Omega$,

$$
\liminf _{x \rightarrow x_{0}} u(x) \geq \varphi\left(x_{0}\right)
$$

On the other hand,

$$
\limsup _{x \rightarrow x_{0}} u(x) \leq \varphi\left(x_{0}\right)
$$

Indeed, for some $R>0$, let $\Omega \subset B_{R}(0)$, we consider

$$
\begin{cases}\Delta v^{+}=0 & \text { in } B_{R}(0) \backslash \bar{\Omega} \\ v^{+}=\varphi & \text { on } \partial \Omega \\ v^{+}=u & \text { on } \partial B_{R}(0) .\end{cases}
$$

Then by the comparison principle for any $v \in \mathbb{S}_{\tilde{d}}, v \leq v^{+}$in $B_{R}(0) \backslash \Omega$, so $u \leq v^{+}$in $B_{R}(0) \backslash \Omega$, and then

$$
\limsup _{x \rightarrow x_{0}} u(x) \leq v^{+}\left(x_{0}\right)=\varphi\left(x_{0}\right), \quad x_{0} \in \partial \Omega
$$

Combining with (3.12), we deduce that

$$
u=\varphi \quad \text { on } \partial \Omega .
$$

In the following, we prove that $u$ is a viscosity solution of (1.14).

For any $\hat{x} \in \mathbb{R}^{2}$, choose $B_{\epsilon}(\hat{x}):=\{x:|x-\hat{x}| \leq \epsilon\} \subset \mathbb{R}^{2} \backslash \bar{\Omega}$. Consider the Dirichlet problem

$$
\begin{aligned}
& \operatorname{det} D^{2} \hat{u}=f \quad \text { in } B_{\epsilon}(\hat{x}), \\
& \hat{u}=u \quad \text { on } \partial B_{\epsilon}(\hat{x}) .
\end{aligned}
$$

By virtue of the comparison principle, we know

$$
u \leq \hat{u} \leq \bar{u}_{\lambda_{2}} \quad \text { in } \overline{B_{\epsilon}(\hat{x})}
$$

Let

$$
\hat{w}(x)= \begin{cases}\hat{u}(x) & \text { in } B_{\epsilon}(\hat{x}), \\ u(x) & \text { in } \mathbb{R}^{2} \backslash\left(\Omega \cup B_{\epsilon}(\hat{x})\right) .\end{cases}
$$


Then $\operatorname{det} D^{2} \hat{w} \geq f$ in $\mathbb{R}^{2} \backslash \bar{\Omega}$ and $\hat{w}(x) \leq \bar{u}_{\lambda_{2}}(x), x \in \mathbb{R}^{2} \backslash \Omega$. Therefore $\hat{w} \in \mathbb{S}_{\tilde{d}}$. And then $u(x) \geq \hat{w}(x), x \in \mathbb{R}^{2} \backslash \Omega$. Especially,

$$
u(x) \geq \hat{w}(x)=\hat{u}(x), \quad x \in B_{\epsilon}(\hat{x}) .
$$

So, by (3.13), we can get

$$
u(x)=\hat{u}(x), \quad x \in B_{\epsilon}(\hat{x})
$$

Since $\hat{x}$ is arbitrary, we know that $u$ is a solution of (1.14).

The uniqueness can be obtained by the comparison principle for viscosity solutions. Theorem 1.6 is proved.

\section{Acknowledgements}

The author is very grateful to the referees for their very helpful comments and suggestions.

\section{Funding}

The research was supported by the National Natural Science Foundation of China (No. 11201343) and the Shandong Provincial Natural Science Foundation (ZR2018LA006).

\section{Availability of data and materials}

Not applicable.

\section{Competing interests}

The author declares that they have no competing interests.

\section{Authors' contributions}

The author contributed completely to the writing of this paper. The author read and approved the final manuscript.

\section{Publisher's Note}

Springer Nature remains neutral with regard to jurisdictional claims in published maps and institutional affiliations.

Received: 19 August 2020 Accepted: 1 December 2020 Published online: 09 December 2020

\section{References}

1. Jörgens, K.: Über die Lösungen der Differentialgleichung $r t-s^{2}=1$. Math. Ann. 127, 130-134 (1954) (German)

2. Calabi, E.: Improper affine hyperspheres of convex type and a generalization of a theorem by K. Jörgens. Mich. Math. J. 5, 105-126 (1958)

3. Pogorelov, A.: On the improper convex affine hyperspheres. Geom. Dedic. 1, 33-46 (1972)

4. Cheng, S.Y., Yau, S.T.: Complete affine hypersurfaces, I. The completeness of affine metrics. Commun. Pure Appl. Math. 39, 839-866 (1986)

5. Caffarelli, L.: Topics in PDEs: the Monge-Ampère equation. Graduate course. Courant Institute, New York University (1995)

6. Jost, J., Xin, Y.L.: Some aspects of the global geometry of entire space-like submanifolds. Dedicated to Shiing-Shen Chern on his 90th birthday. Results Math. 40, 233-245 (2001)

7. Trudinger, N.S., Wang, X.J.: The Bernstein problem for affine maximal hypersurfaces. Invent. Math. 140, 399-422 (2000)

8. Caffarelli, L., Li, Y.Y.: An extension to a theorem of Jörgens, Calabi, and Pogorelov. Commun. Pure Appl. Math. 56 549-583 (2003)

9. Ferrer, L., Martínez, A., Milán, F.: An extension of a theorem by K. Jörgens and a maximum principle at infinity for parabolic affine spheres. Math. Z. 230, 471-486 (1999)

10. Ferrer, L., Martínez, A., Milán, F.: The space of parabolic affine spheres with fixed compact boundary. Monatshefte Math. 130, 19-27 (2000)

11. Delanoë, P.: Partial decay on simple manifolds. Ann. Glob. Anal. Geom. 10, 3-61 (1992)

12. Caffarelli, L., Li, Y.Y.: A Liouville theorem for solutions of the Monge-Ampère equation with periodic data. Ann. Inst. Henri Poincaré, Anal. Non Linéaire 21, 97-120 (2004)

13. Bao, J.G., Li, H.G., Zhang, L.: Monge-Ampère equation on exterior domains. Calc. Var. Partial Differ. Equ. 52, 39-63 (2015)

14. Bao, J.G., Li, H.G.: On the exterior Dirichlet problem for the Monge-Ampère equation in dimension two. Nonlinear Anal. 75, 6448-6455 (2012)

15. Bao, J.G., Li, H.G., Zhang, L.: Global solutions and exterior Dirichlet problem for Monge-Ampère equation in $\mathbb{R}^{2}$. Differ Integral Equ. 29, 563-582 (2016) 
16. Bao, J.G., Xiong, J.G., Zhou, Z.W.: Existence of entire solutions of Monge-Ampère equations with prescribed asymptotic behavior. Calc. Var. Partial Differ. Equ. 58, Paper No. 193 (2019)

17. Ju, H.J., Bao, J.G.: On the exterior Dirichlet problem for Monge-Ampère equations. J. Math. Anal. Appl. 405, 475-483 (2013)

18. Wang, C., Bao, J.G.: Necessary and sufficient conditions on existence and convexity of solutions for Dirichlet problems of Hessian equations on exterior domains. Proc. Am. Math. Soc. 141, 1289-1296 (2013)

19. Li, Y.Y., Lu, S.Y.: Existence and nonexistence to exterior Dirichlet problem for Monge-Ampère equation. Calc. Var. Partial Differ. Equ. 57, Paper No. 161 (2018)

20. Mohammed, A., Radulescu, V.D., Vitolo, A.: Blow-up solutions for fully nonlinear equations: existence, asymptotic estimates and uniqueness. Adv. Nonlinear Anal. 9(1), 39-64 (2020)

21. Zhang, X.M., Feng, M.Q.: Boundary blow-up solutions to the Monge-Ampère equation: sharp conditions and asymptotic behavior. Adv. Nonlinear Anal. 9(1), 729-744 (2020)

Submit your manuscript to a SpringerOpen ${ }^{\odot}$ journal and benefit from:

- Convenient online submission

- Rigorous peer review

- Open access: articles freely available online

- High visibility within the field

- Retaining the copyright to your article

Submit your next manuscript at $\gg$ springeropen.com 